\title{
Suppression of Cross-Polarization of the Microstrip Integrated Balun-Fed Printed Dipole Antenna
}

\author{
Huang Jingjian, Zhang Xiaofa, Xie Shaoyi, Wu Weiwei, and Yuan Naichang \\ College of Electronic Science and Engineering, National University of Defense Technology, Changsha 410073, China \\ Correspondence should be addressed to Huang Jingjian; hjjfh2003@aliyun.com
}

Received 25 December 2013; Accepted 24 February 2014; Published 1 April 2014

Academic Editor: Xiuping Li

Copyright (C) 2014 Huang Jingjian et al. This is an open access article distributed under the Creative Commons Attribution License, which permits unrestricted use, distribution, and reproduction in any medium, provided the original work is properly cited.

\begin{abstract}
The high cross-polarization of the microstrip integrated balun-fed printed dipole antenna cannot meet the demands of many engineering applications. This kind of antennas has high cross-polarization levels (about $-20 \mathrm{~dB}$ ). And we find that the high crosspolarization radiation is mainly produced by the microstrip integrated balun rather than the dipole itself. The very limited method to lower the cross-polarization level of this kind of antennas is to reduce the substrate thickness. In this paper, to improve the low cross-polarized performance, firstly, an equivalent model is presented to analyze the cross-polarization radiation. Secondly, a novel structure with low cross-polarization is proposed. The microstrip integrated balun is enclosed by a center slotted cavity. The E-field of the microstrip integrated balun is transformed parallel to the dipole arms by the slot, so the radiation of the cross-polarized component is suppressed. Measured results show that this structure can achieve a bandwidth wider than $40 \%$ while reducing the cross-polarization level to less than $-35 \mathrm{~dB}$ within the frequency band.
\end{abstract}

\section{Introduction}

Microstrip integrated balun-fed printed dipole antenna (PDA) is proposed by Edward and Rees [1] in 1987, and it is widely used in the communication and radar systems [1-12], owing to their salient features such as light weight, wideband, low cost and ease of fabrication, and suitability for integration with circuit modules. In 1989, Proudfoot [2] designed a folded printed dipole. The radiation impedance can be adjusted from $70 \mathrm{ohms}$ to $200 \mathrm{ohms}$. And Proudfoot also supposed that the high cross-polarization is caused by the microstrip integrated balun rather than the dipole itself. Now, many applications require low crosspolarization. Zhou et al. [12] proposed a double-layered structure to improve the cross-polarization. But its stripline feeding structure made it hard to feed by a coaxial connector.

In this paper, an equivalent model to analyze the crosspolarization of the microstrip integrated balun-fed PDA is presented. Based on the equivalent circuit [13] of the microstrip integrated balun, an impedance element is placed in the center of the slotted balun to replace the dipole radiation impedance and fill the slot of the shorting slotline between the two dipole arms with metal to restrain the radiation of the dipole. The E-plane and H-plane crosspolarized patterns are compared. The accordant results validate that the cross-polarization is mainly radiated by the microstrip integrated balun rather than the dipole itself. Then the cross-polarization varying with the main parameters of the substrate is studied. The simulated results show that it has very limited improvement on the cross-polarization by varying the substrate parameters. To improve the crosspolarization performance, a printed dipole antenna whose microstrip integrated balun is enclosed by a center slotted cavity is proposed. The microstrip integrated balun is moved down [3], so the input impedance can be directly matched to $50 \mathrm{ohm}$. Furthermore, the cavity can be placed under the dipole arms. This structure can create the wide bandwidth characteristic while considerably suppressing the cross-polarized component. The antenna is simulated by Ansoft Corporation's high frequency structure simulator (HFSS). 


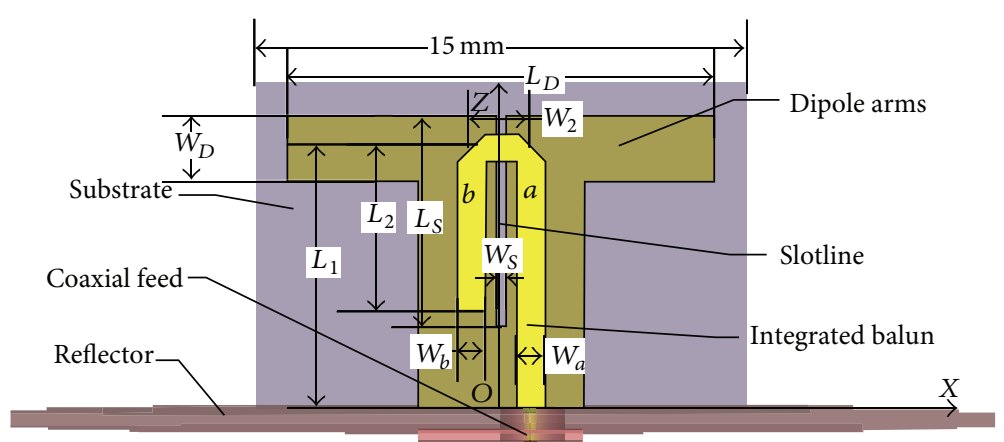

(a) Front view

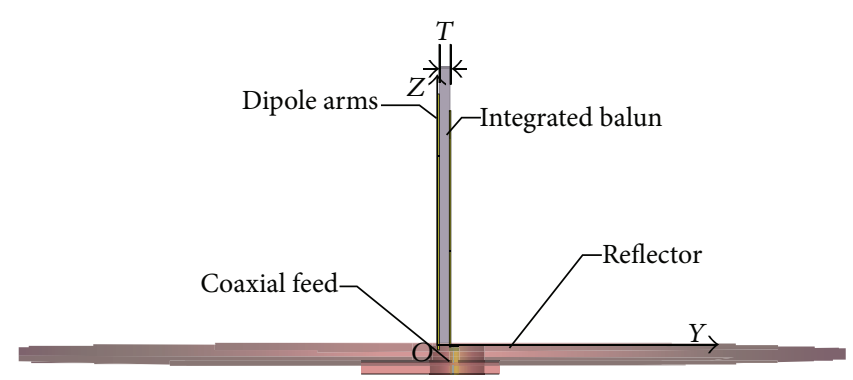

(b) Side view

FIGURE 1: Geometry of the microstrip integrated balun-fed PDA.

\section{Cross-Polarization Analysis}

Due to the asymmetrical feeding structure, the $Y$ directional E-field component could be activated between the microstrip integrated balun and the dipole arms. This part of electronic field can make a high level cross-polarization.

The geometry of the microstrip integrated balun-fed PDA is shown in Figure 1. The front view and side view of this antenna model are shown in Figure 1(a) and Figure 1(b), respectively. Microstrip integrated balun and dipole arms are printed on each side of the substrate. The antenna is placed on a circular reflector with a diameter of $30 \mathrm{~mm}$ and fed by a coaxial connector. The microstrip integrated balun has a microstrip line " $a$ " with width $W_{a}=0.87 \mathrm{~mm}$, a microstrip line " $b$ " with width $W_{b}=0.87 \mathrm{~mm}$, the balun with width $W_{2}=$ $1.8 \mathrm{~mm}$ and length $L_{1}=7.5 \mathrm{~mm}$, and match branch with length $L_{2}=5 \mathrm{~mm}$. The substrate has the relative permittivity er $=2.2$ and thickness $T=0.5 \mathrm{~mm}$. The dipole has slotline width $W_{S}=0.3 \mathrm{~mm}$ and slotline length $L_{S}=6.83 \mathrm{~mm}$ and dipole arm width $W_{D}=2 \mathrm{~mm}$ and length $L_{D}=13 \mathrm{~mm}$.

The equivalent circuit is shown in Figure 2. According to the transmission line theory, the input impedance can be written as

$$
Z_{\text {in }}=-j Z_{b} \cot \theta_{b}+\frac{J Z_{L} Z_{s} \tan \theta_{s}}{Z_{L}+j Z_{s} \tan \theta_{s}} .
$$

When the input impedance $Z_{\text {in }}$ is equal to the characteristic impedance $Z_{a}$ of the microstrip line " $a$ ", the feeding network can be well matched. We set the input impedance $Z_{\text {in }}$ equal to the antenna radiation impedance $Z_{L}$. So the length of the shorting slotline $\theta_{s}$ and the length of " $b$ " segment $\theta_{b}$ are

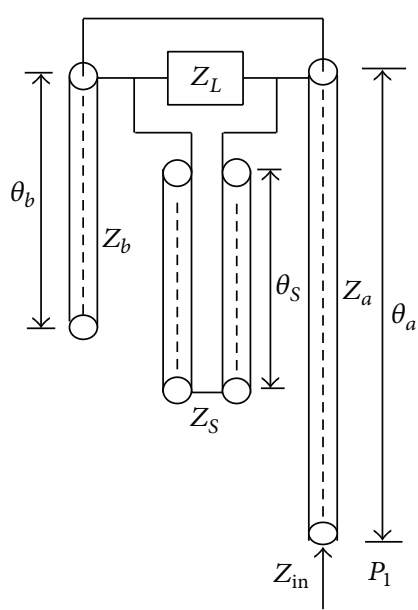

FIGURE 2: The equivalent circuit of the microstrip integrated balun.

both setting to a quarter waveguide wavelength. So formula (1) can be rewritten as

$$
Z_{\text {in }}=Z_{L}
$$

The current in " $a$ " segment of the integrated balun is a pure travelling wave, and the current in " $b$ " segment is a standing wave. So we can suppose that the cross-polarization radiation of this antenna is mainly produced by the sum of the cross-polarized radiation of the " $a$ " segment and the " $b$ " segment.

To verify that the cross-polarization is mainly radiated by the microstrip integrated balun, the radiation of the dipole needs to be restrained. Meanwhile, the current distribution 


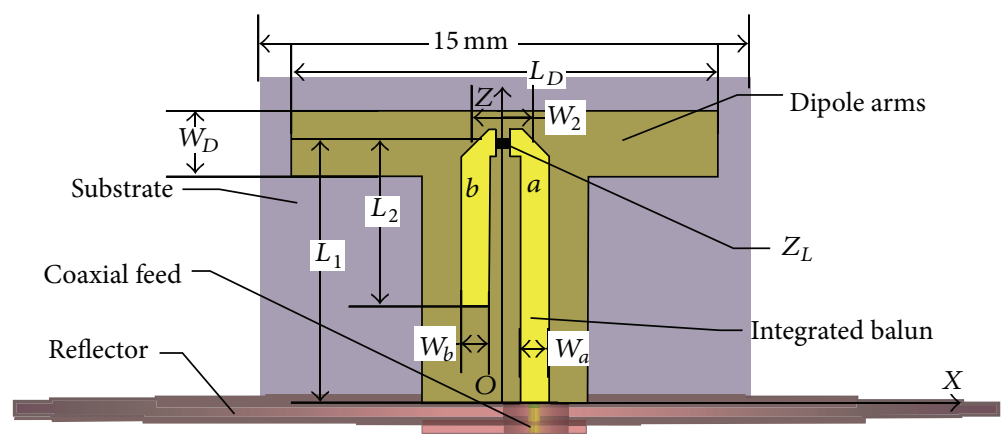

FIGURE 3: Geometry of the modified model.

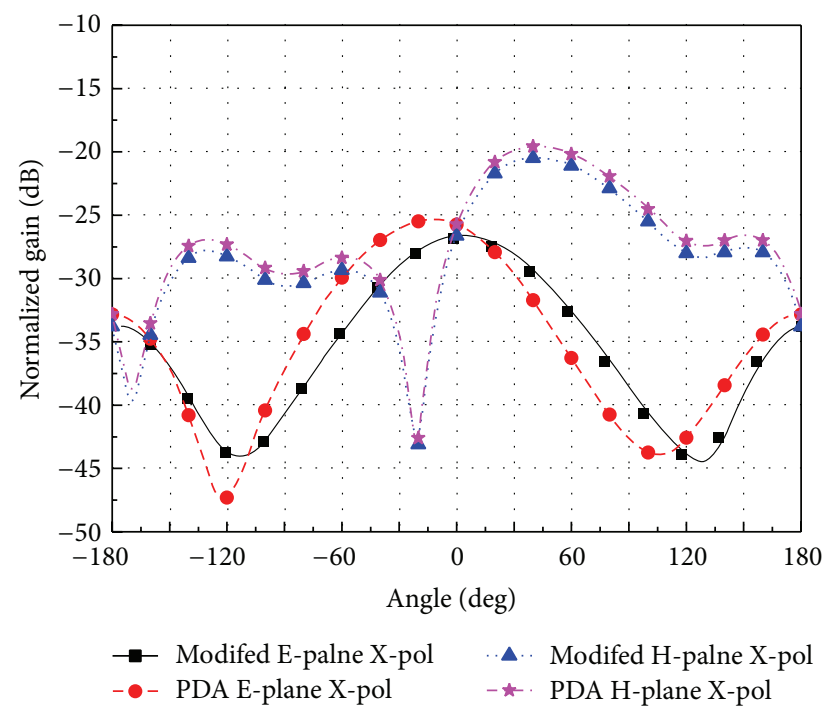

FIGURE 4: Cross-polarization radiation pattern of the conventional PDA and modified model at $10 \mathrm{GHz}$.

of the microstrip integrated balun should be well remained. Firstly, the shorting slotline is filled with metal. So the radiation from dipole is suppressed, and moreover, the boundary condition can be well maintained. Secondly, a narrow slot is cut at the middle of the microstrip integrated balun, and then an impedance element with impedance $Z_{L}$ is placed in this slot. According to the equivalent circuit analysis above, the current distribution in the integrated balun will be maintained. The geometry of the modified model is shown in Figure 3.

The $\mathrm{H}$-plane and E-plane cross-polarization radiation patterns of the conventional PDA and the modified model are compared in Figure 4. It shows that both H-plane crosspolarization and E-plane cross-polarization of the modified model agreed well with the conventional PDAs. Because the coupling effect between the slotline and the integrated balun is considered in the modified model, the crosspolarization levels are a little bit lower than the conventional PDAs. These results prove that the cross-polarization of the microstrip integrated balun-fed PDA is mainly radiated by the microstrip integrated balun.

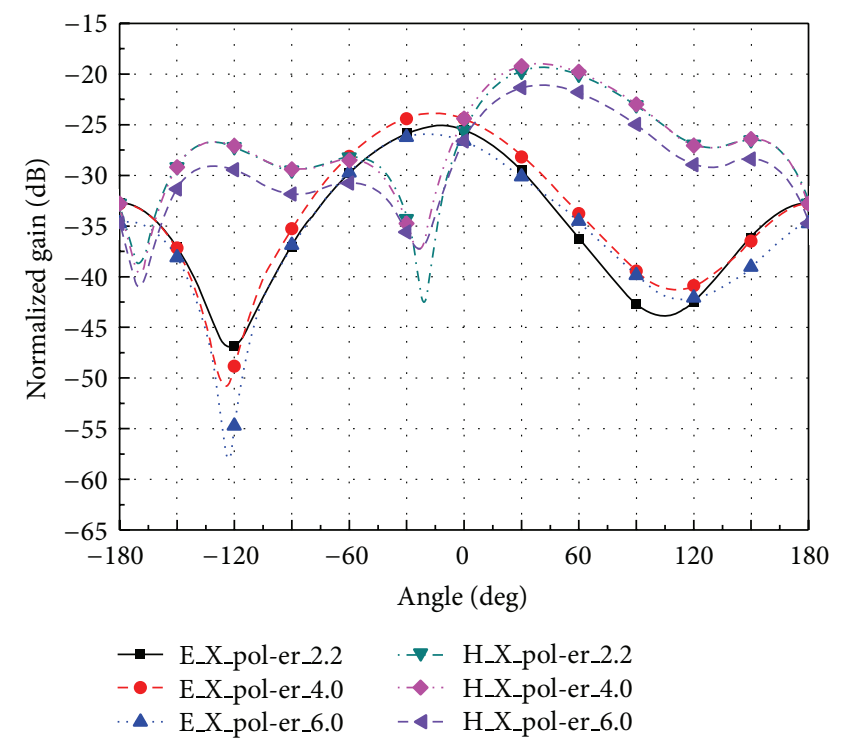

FIGURE 5: Cross-polarization pattern with different relative permittivity.

\section{Cross-Polarization Varies with Substrate Parameters}

To design a PDA, the selection of the substrate is very important. While the parameters of the substrate are decided, the geometry of this antenna can be decided too. For example, when the substrate parameters are obtained, the width and the waveguide wavelength of the microstrip line can be calculated [14] at a given frequency $(10 \mathrm{GHz}$ in this paper). The thickness and the relative permittivity are the two key parameters, so in this section we will analyze the relations between the cross-polarization and these two substrate parameters, respectively.

3.1. Cross-Polarization Varies with the Substrate Relative Permittivity. In Table 1, we keep the substrate thickness $T=$ $0.5 \mathrm{~mm}$, and then some main dimensions are calculated at $10 \mathrm{GHz}$ with different relative permittivity er $(2.2,4.0$, and 6.0). The corresponding cross-polarization far-field patterns are shown in Figure 5. And the cross-polarized levels versus 


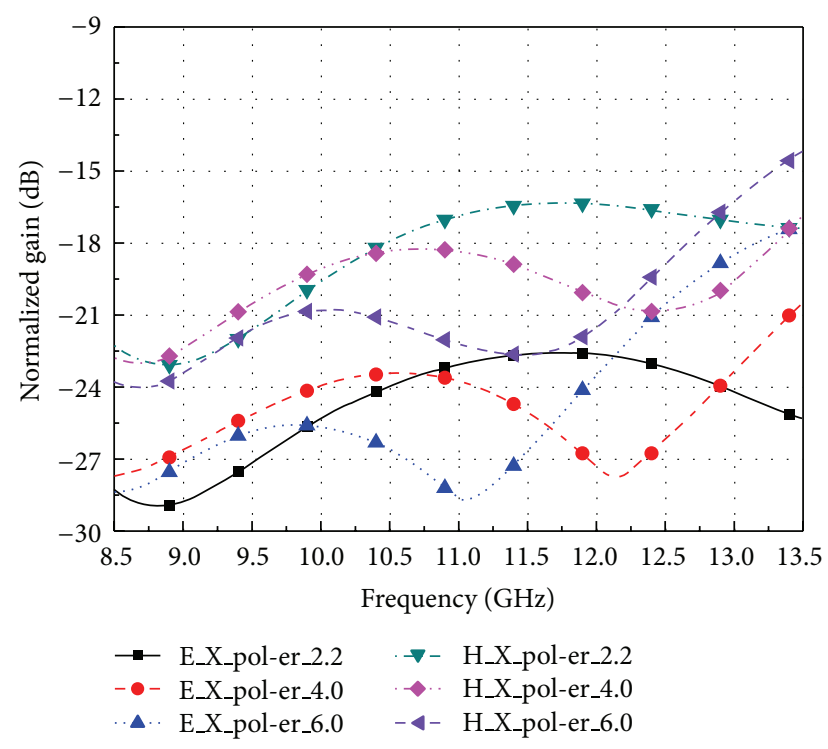

Figure 6: Cross-polarized level versus frequency with different relative permittivity.

TABLE 1: Dimensions vary with the relative permittivity.

\begin{tabular}{lcccc}
\hline $\mathrm{er}$ & $L_{2}(\mathrm{~mm})$ & $W_{a}(\mathrm{~mm})$ & $L_{S}(\mathrm{~mm})$ & $L_{D}(\mathrm{~mm})$ \\
\hline 2.2 & 5.0 & 0.87 & 6.83 & 13.6 \\
4 & 3.9 & 0.54 & 5.88 & 12.6 \\
6 & 3.3 & 0.37 & 5.14 & 11.5 \\
\hline
\end{tabular}

frequency with different relative permittivity are plotted in Figure 6. As we can see, the cross-polarization varies little with the substrate permittivity. And there is no certain relationship between the cross-polarization and the substrate permittivity. The higher relative permittivity can increase the electrical length between the microstrip line and the ground, so more energy can be released. But the higher relative permittivity also reduces the geometrical size of the microstrip line, so the radiation ability will be weakened. Due to these two reasons, the cross-polarization cannot be suppressed by changing the substrate permittivity.

3.2. Cross-Polarization Variations with the Substrate Thickness. In Table 2, we keep the substrate relative permittivity on 2.2 , and then some main dimensions are calculated at $10 \mathrm{GHz}$ with different substrate thicknesses $(0.3 \mathrm{~mm}, 0.5 \mathrm{~mm}$, and $0.7 \mathrm{~mm}$ ). The corresponding cross-polarization far-field patterns are shown in Figure 7, and the cross-polarized levels versus frequency with different substrate thicknesses are plotted in Figure 8. The results show that the crosspolarization levels are reduced while the substrate thickness is thinned. The cross-polarized levels have about $5 \mathrm{~dB}$ increase throughout the band while the substrate thickness varies from $0.3 \mathrm{~mm}$ to $0.7 \mathrm{~mm}$. This is because the thinner the substrate is, the less the energy can be radiated from the microstrip integrated balun. However, the substrate thickness cannot be too thin, or the antenna mechanical performance will be too weak for application.

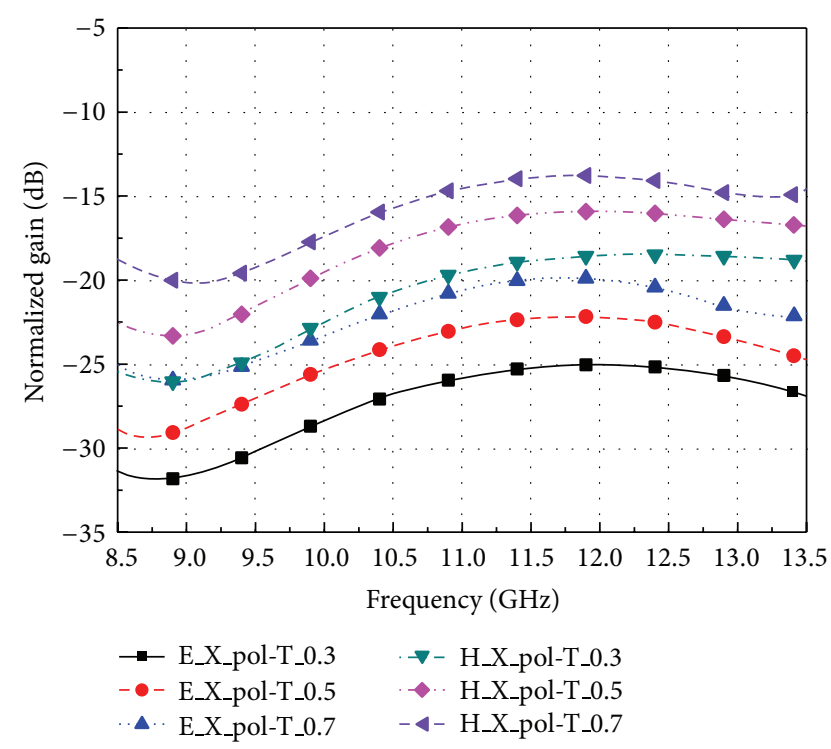

Figure 7: Cross-polarized levels versus frequency with different substrate thicknesses.

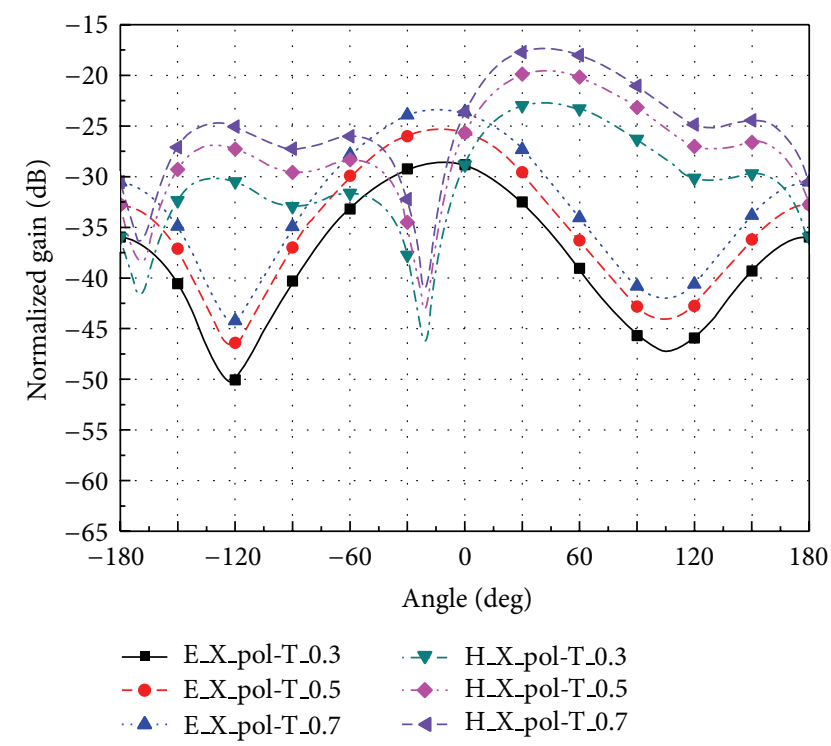

Figure 8: Cross-polarization far-field pattern with different substrate thicknesses.

TABLE 2: Dimensions vary with the substrate thickness.

\begin{tabular}{lcccc}
\hline$T(\mathrm{~mm})$ & $L_{2}(\mathrm{~mm})$ & $W_{a}(\mathrm{~mm})$ & $L_{S}(\mathrm{~mm})$ & $L_{D}(\mathrm{~mm})$ \\
\hline 0.3 & 5.0 & 0.52 & 6.98 & 13.9 \\
0.5 & 5.0 & 0.87 & 6.83 & 13.6 \\
0.7 & 5.0 & 1.24 & 6.75 & 13.4 \\
\hline
\end{tabular}

According to the above study, we can conclude that the cross-polarization of the microstrip integrated balun-fed PDA is mainly radiated from the microstrip integrated balun, and thinning the substrate can reduce the cross-polarization level. But the suppression of the cross-polarization is very limited. 


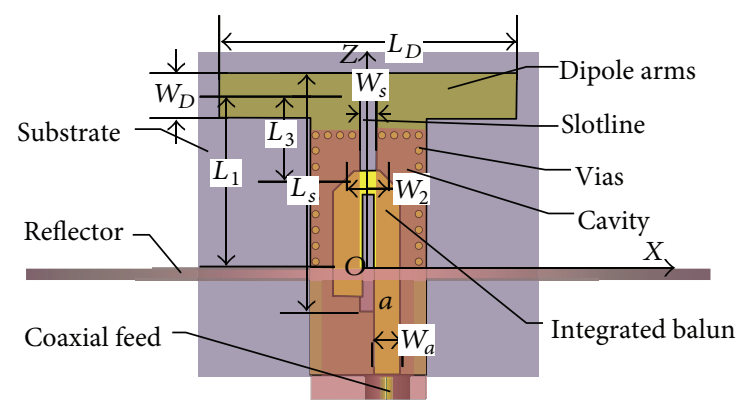

(a) Front view

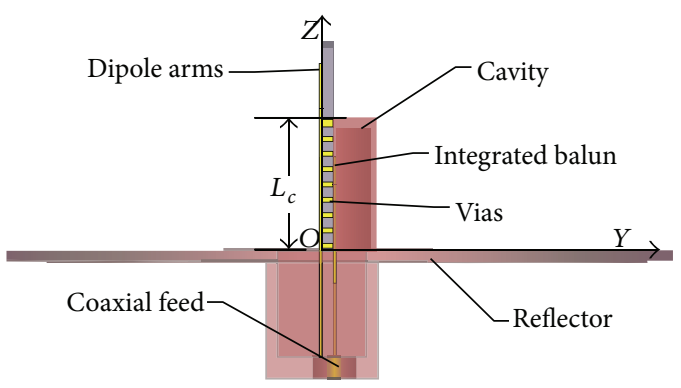

(b) Back view

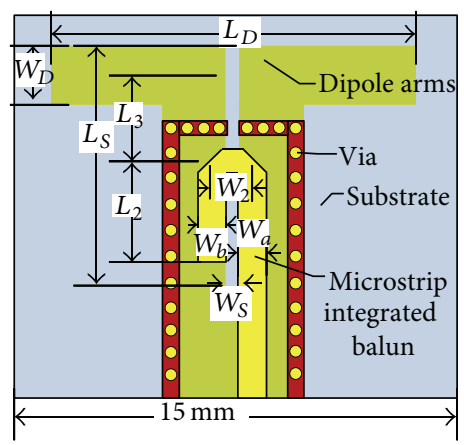

(c) Geometry of the printed structure

FIgURE 9: Antenna configuration.

\section{Configuration of the Improved Antenna}

In this section we propose a novel structure to greatly suppress the cross-polarization. The improved structure is shown in Figure 9. Figures 9(a) and 9(b) show the front view and side view of the antenna, respectively. Figure 9(c) shows the geometry of the printed dipole. The microstrip integrated balun is moved down $L_{3}$. The microstrip integrated balun is enclosed by a slotted cavity with a height $L_{c}$. This cavity is formed by a semicylinder, a series of vias, and the ground of the microstrip integrated balun. The vias are closed to each other. So the EM-field in the working band cannot leak from the substrate side. The width of the slotline is equal to the width of the shorting line. So the radiation EM-field of the microstrip integrated balun is transformed by these slots. The E-field on the slot is parallel to the dipole arms, so the radiation polarization direction of the slot is parallel to the main polarization direction of the dipole. And the cross-polarization could be greatly suppressed. The attractive

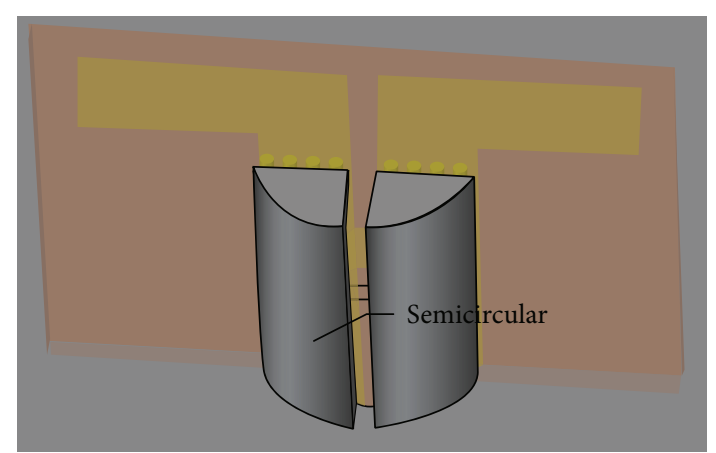

(a)

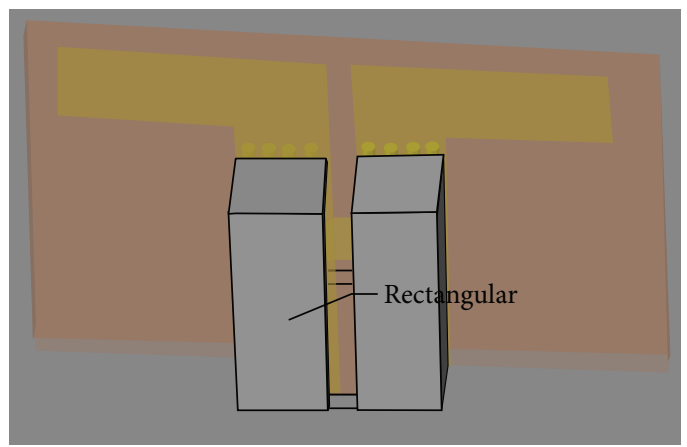

(b)

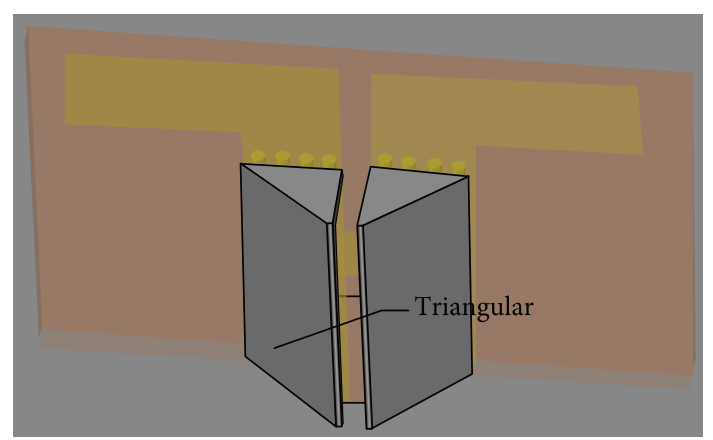

(c)

FIGURE 10: Three different kinds of slotted cavities.

features of this proposed structure will be verified by the following simulations and measurements.

The cross-polarized levels of three different slotted cavities, as shown in Figure 10 (semicircular, rectangular, and triangular), are compared. The results are shown in Figure 11. It shows that the gains throughout the band are the same. And compared to the conventional PDA (analyzed in foregoing section), all these results have lower cross-polarized levels. Figure 11 also shows that the triangular and semicircular cavities have better cross-polarized level than the rectangular one. This is due to the fact that the triangular and semicircular cavities have taper boundary condition, and such boundaries have smaller coupling effects with the dipole arms than the rectangular boundary. Then the $Y$-directional E-field is weaker. So the cross-polarization component can be greatly suppressed. 


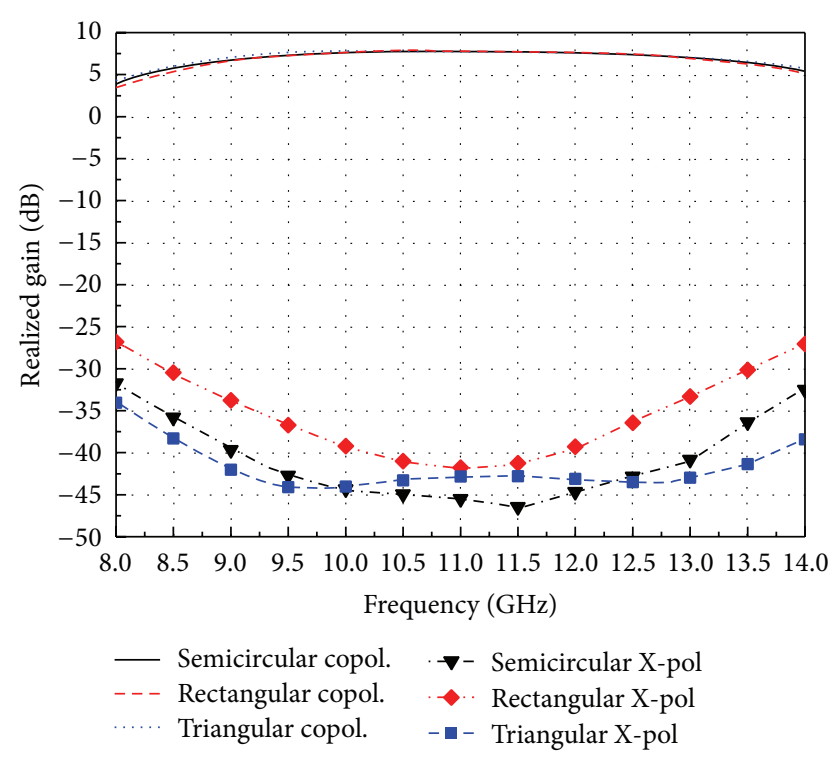

FIGURE 11: Main polarized levels and cross-polarized levels versus frequency with different slotted cavities.

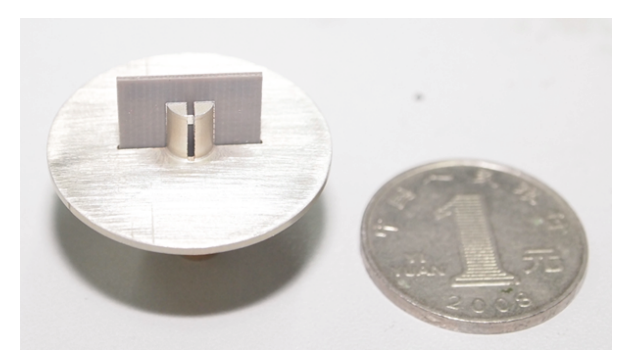

(a)

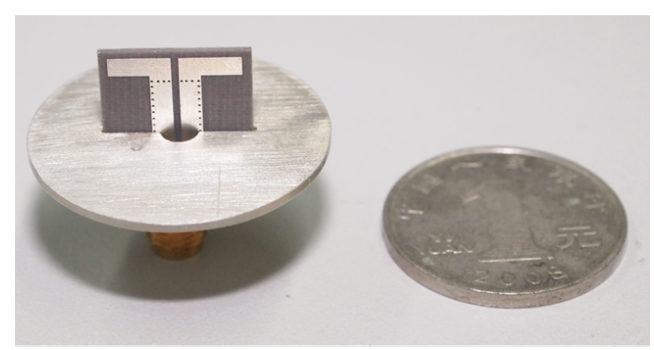

(b)

FIGURE 12: Photo of the fabricated antenna.

\section{Fabrication and Test}

This improved antenna is fabricated, as shown in Figure 12. The dipole arms and balun are printed on a dielectric substrate with relative permittivity er $=2.2$, thickness $T=$ $0.5 \mathrm{~mm}$, and loss tangent of 0.001 . The circular ground plane with a diameter of $30 \mathrm{~mm}$ is placed below the dipole arms. And some main dimensions are listed as follows: $L_{D}=$ $13 \mathrm{~mm}, W_{D}=2 \mathrm{~mm}, L_{S}=10.5 \mathrm{~mm}, W_{S}=0.7 \mathrm{~mm}, W_{a}=$ $1.2 \mathrm{~mm}, W_{2}=1.8 \mathrm{~mm}, L_{1}=8.0 \mathrm{~mm}, L_{3}=4 \mathrm{~mm}$, and $L_{C}=6.5 \mathrm{~mm}$.

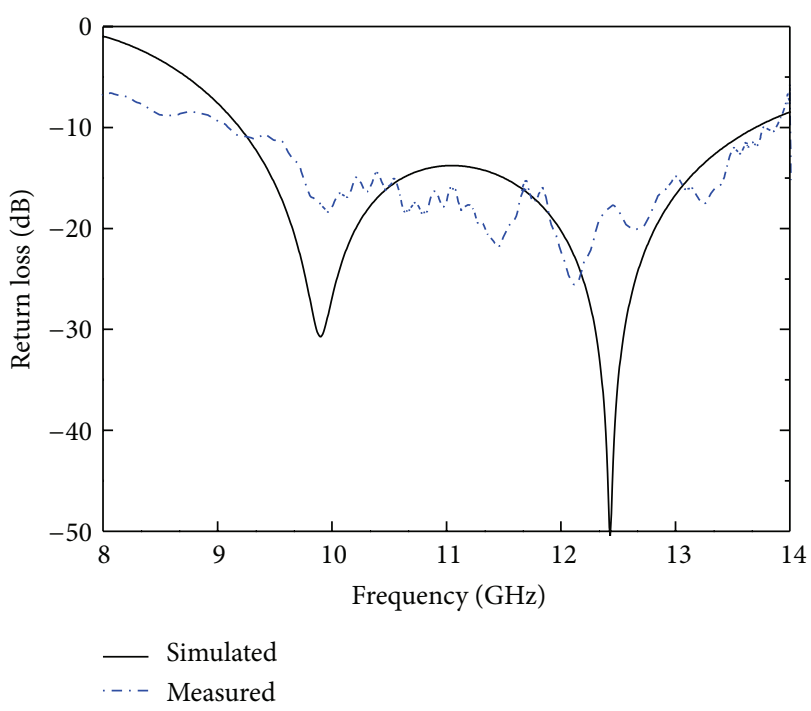

FIGURE 13: Return loss versus frequency.

Figure 13 shows the simulated and measured return loss in solid line and dashed line, respectively. The results show that $-10 \mathrm{~dB}$ return loss bandwidths are wider than $40 \%$. It is observed that this improved structure can maintain the wide bandwidth feature.

The E-plane and $\mathrm{H}$-plane radiation patterns at $10 \mathrm{GHz}$ are plotted in Figures 14(a) and 14(b), respectively. It is observed that the measured copolarized radiation patterns in both Eplane and $\mathrm{H}$-plane agreed well with the simulated results. But the measured E-plane and H-plane cross-polarized pattern are about $5 \mathrm{~dB}$ and $18 \mathrm{~dB}$ higher than the simulated result, respectively. This is because the cross-polarized level probably reaches to the lower limit of the measured system.

Figure 15 plots the realized gain and the crosspolarization levels (the maximum cross-polarization level of the E-plane and H-plane in the beam is between $-90^{\circ}$ and $90^{\circ}$ ) versus frequency. It shows that both simulated and measured results have even wider $3 \mathrm{~dB}$ realized gain bandwidth than the $-10 \mathrm{~dB}$ return loss bandwidth. The tested cross-polarization level is almost below $-35 \mathrm{~dB}$ at the band (8.5-13.5 GHz).

\section{Conclusion}

An equivalent model for analyzing the cross-polarization of the microstrip integrated balun-fed PDA is presented. And the cross-polarizations of the equivalent model and the conventional model are compared. The E-plane and H-plane cross-polarization patterns agreed well with the conventional one. It is demonstrated that the cross-polarization is mainly radiated from the microstrip integrated balun rather than the dipole itself. The cross-polarization varying with the substrate parameters of thicknesses and relative permittivity is analyzed. The results show that the cross-polarized level can be reduced by thinning the substrate, but the crosspolarization suppression is very limited. And the variation 


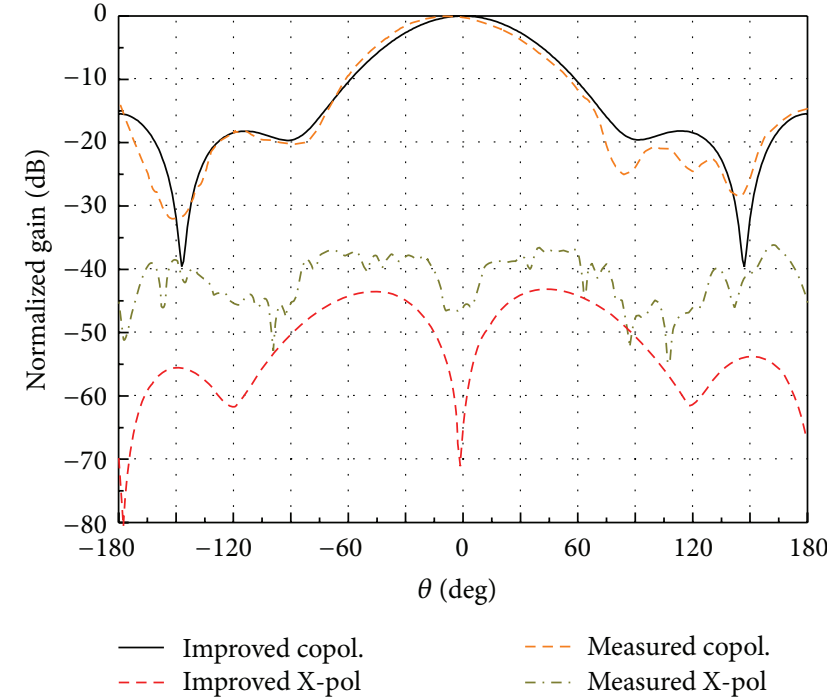

(a) E-plane

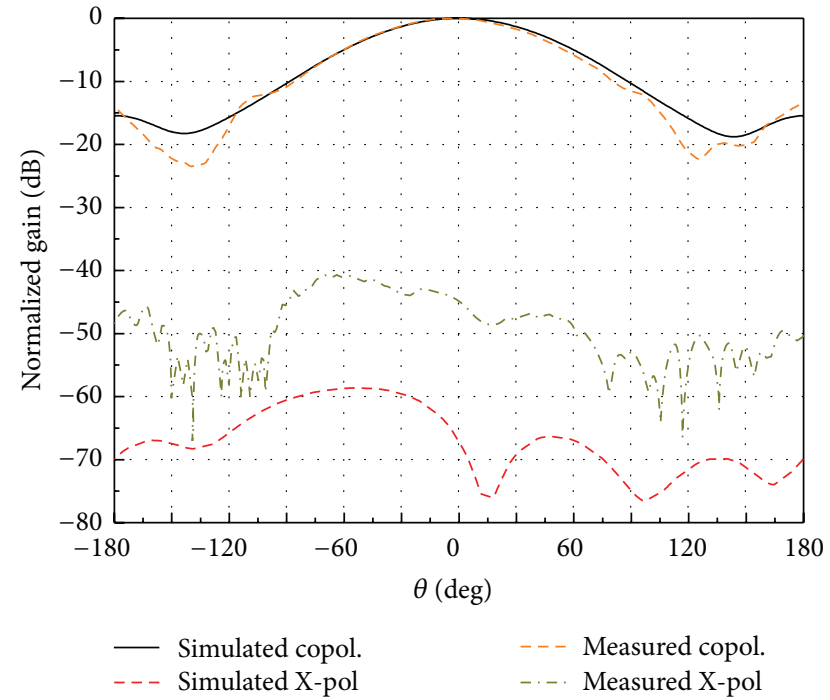

(b) H-plane

FIGURE 14: Far-field pattern at $10 \mathrm{GHz}$.

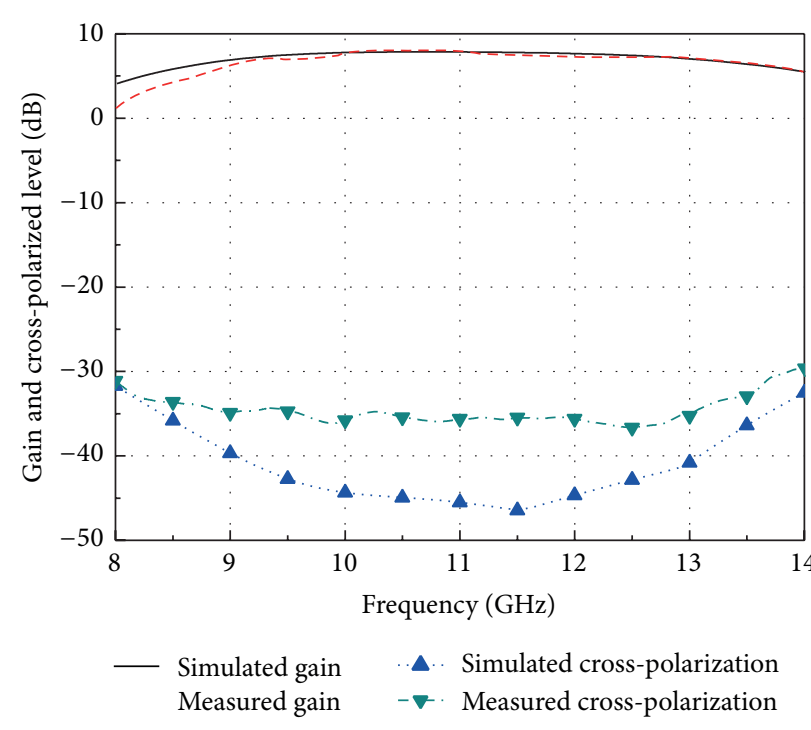

FIGURE 15: Realized gain and the cross-polarization level versus frequency.

of the substrate permittivity has few effects on the crosspolarization. In order to meet the low cross-polarization demands of some applications, such as the low sidelobe arrays and dual polarized arrays, an improved printed dipole element is designed. The microstrip integrated balun is enclosed by a center slotted cavity. And three different kinds of slotted cavities are compared. The simulated results show that the taper boundary (triangular or semicircular) can possess better low cross-polarized performance. An antenna with semicircular cavity is fabricated and tested. The simulated and measured results are compared. It achieves a relative bandwidth wider than $40 \%$ and a cross-polarization level lower than $-35 \mathrm{~dB}$ within the band. The results show that this novel structure can maintain the wideband characteristic while the cross-polarization is restrained. This improved microstrip integrated balun-fed PDA can be easily extended to a large array and is suitable for the applications with a low cross-polarization requirement.

\section{Conflict of Interests}

The authors have no conflict of interests.

\section{Acknowledgment}

This paper is partially supported by the National Science Foundation of China (Grant no. 61302017).

\section{References}

[1] B. Edward and D. Rees, "A broadband printed dipole with integrated balun," Microwave Journal, vol. 30, no. 5, pp. 339-344, 1987.

[2] P. M. Proudfoot, "A printed circuit folded dipole with integrated balun," In-House Report RADC-TR-89-237, 1989.

[3] R. L. Li, T. Wu, B. Pan, K. Lim, J. Laskar, and M. M. Tentzeris, "Equivalent-circuit analysis of a broadband printed dipole with adjusted Integrated balun and an array for base station applications," IEEE Transactions on Antennas and Propagation, vol. 57, no. 7, pp. 2180-2184, 2009.

[4] Y. Cai, Y. J. Guo, and P.-Y. Qin, "Frequency switchable printed Yagi-Uda dipole sub-array for base station antennas," IEEE Transactions on Antennas and Propagation, vol. 60, no. 3, pp. 1639-1642, 2012.

[5] P. Lindberg, E. Ojefors, Z. Barna, A. Thornell-Pers, and A. Rydberg, "Dual wideband printed dipole antenna with integrated balun," IET Microwaves, Antennas \& Propagation, vol. 1, no. 3, pp. 707-711, 2007. 
[6] A. Vallecchi, J. R. de Luis, F. Capolino, and F. de Flaviis, "Low profile fully planar folded dipole antenna on a high impedance surface," IEEE Transactions on Antennas and Propagation, vol. 60, no. 1, pp. 51-62, 2012.

[7] Y. Wen, Z. H. Xue, S. M. Yang, W. Ren, and W. M. Li, "Design and analysis of a $3 \mathrm{GHz}$ printed dipole antenna," in Proceedings of the 4th International Conference on Microwave and Millimeter Wave Technology (ICMMT '04), pp. 8-11, Nanjing, China, August 2004.

[8] Z. G. Fan, S. Qiao, J. T. Huangfu, and L. X. Ran, "A miniaturized printed dipole antenna with V-shaped ground for $2.45 \mathrm{GHz}$ RFID readers," Progress in Electromagnetics Research, vol. 71, pp. 149-158, 2007.

[9] M. Scott, "A printed dipole for wide-scanning array application," in Proceedings of the 11th International Conference on Antenna and Propagation, vol. 1, no. 480, pp. 37-40, Manchester, UK, 2001.

[10] K.-L. Wong, F.-R. Hsiao, and C.-L. Tang, "A low-profile omnidirectional circularly polarized antenna for WLAN access point," in Proceedings of the IEEE Antennas and Propagation Society Symposium, vol. 3, pp. 2580-2583, Monterey, Calif, USA, June 2004.

[11] H.-R. Chuang and L.-C. Kuo, "3-D FDTD design analysis of a 2.4-GHz polarization-diversity printed dipole antenna with integrated balun and polarization-switching circuit for WLAN and wireless communication applications," IEEE Transactions on Microwave Theory and Techniques, vol. 51, no. 2, pp. 374-381, 2003.

[12] Z.-W. Zhou, S.-W. Yang, and Z.-P. Nie, "A novel broadband printed dipole antenna with low cross-polarization," IEEE Transactions on Antennas and Propagation, vol. 55, no. 11, pp. 3091-3093, 2007.

[13] W. K. Roberts, "A new wide-band balun," Proceedings of the IRE, vol. 45, no. 12, pp. 1628-1631, 1957.

[14] R. K. Hoffman, Handbook of Microwave Integrated Circuits, Artech House, Norwood, Mass, USA, 1987. 

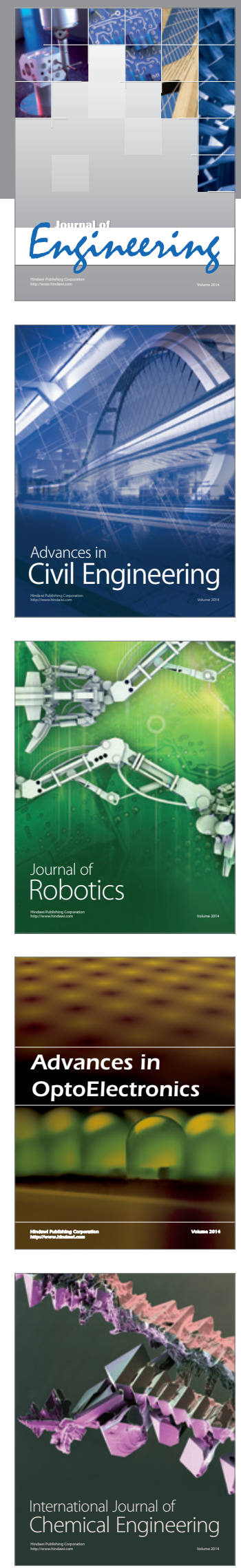

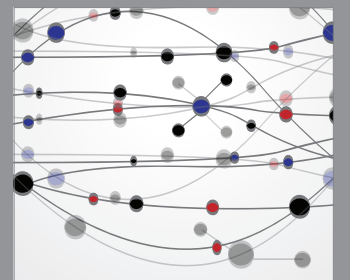

The Scientific World Journal
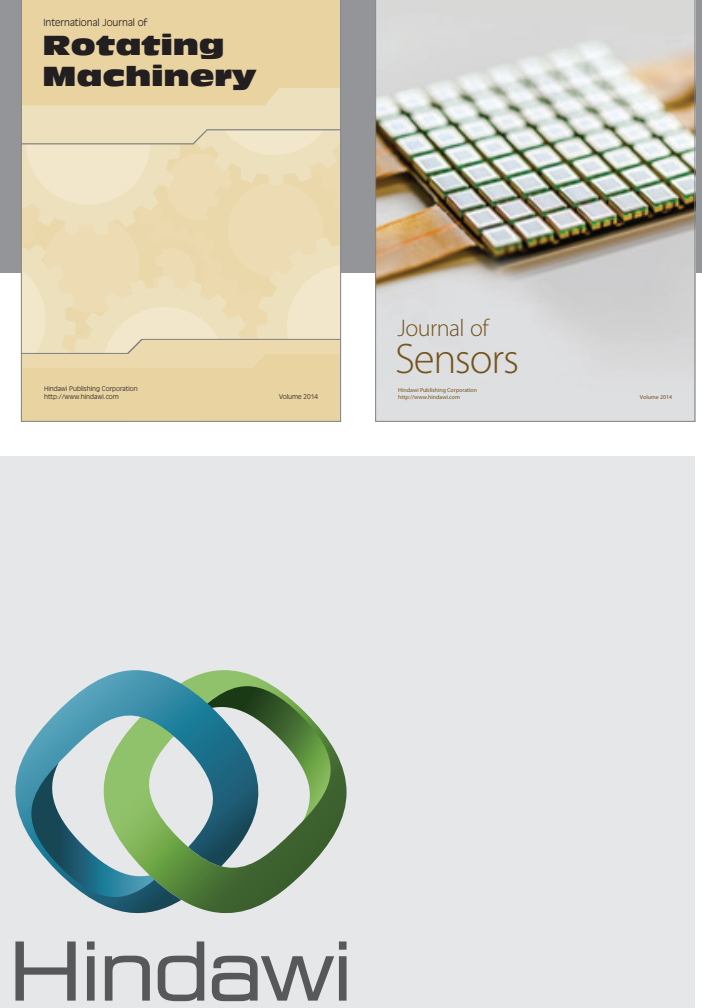

Submit your manuscripts at http://www.hindawi.com
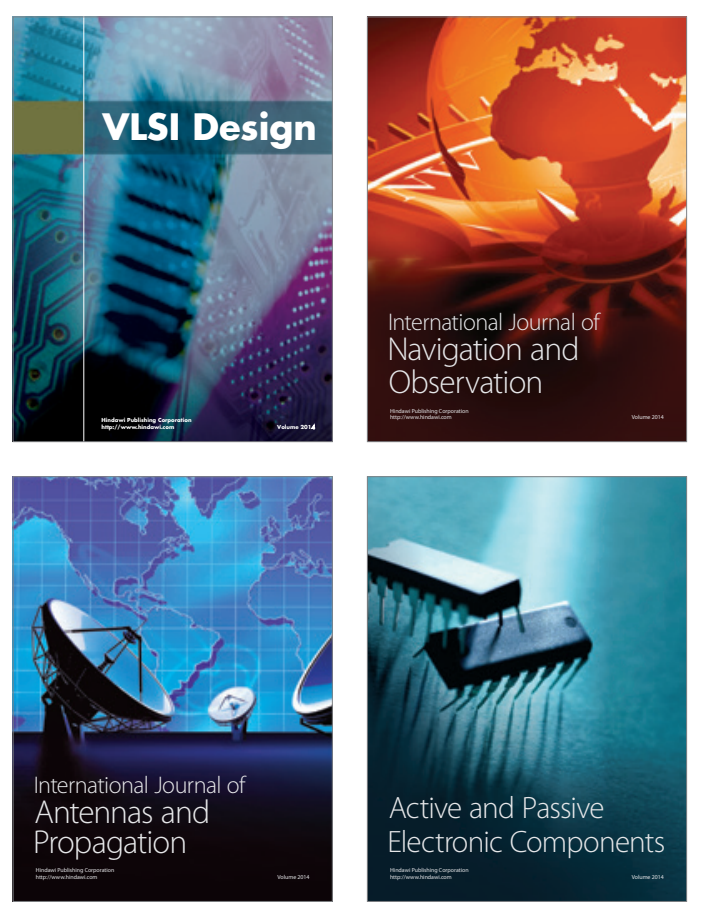
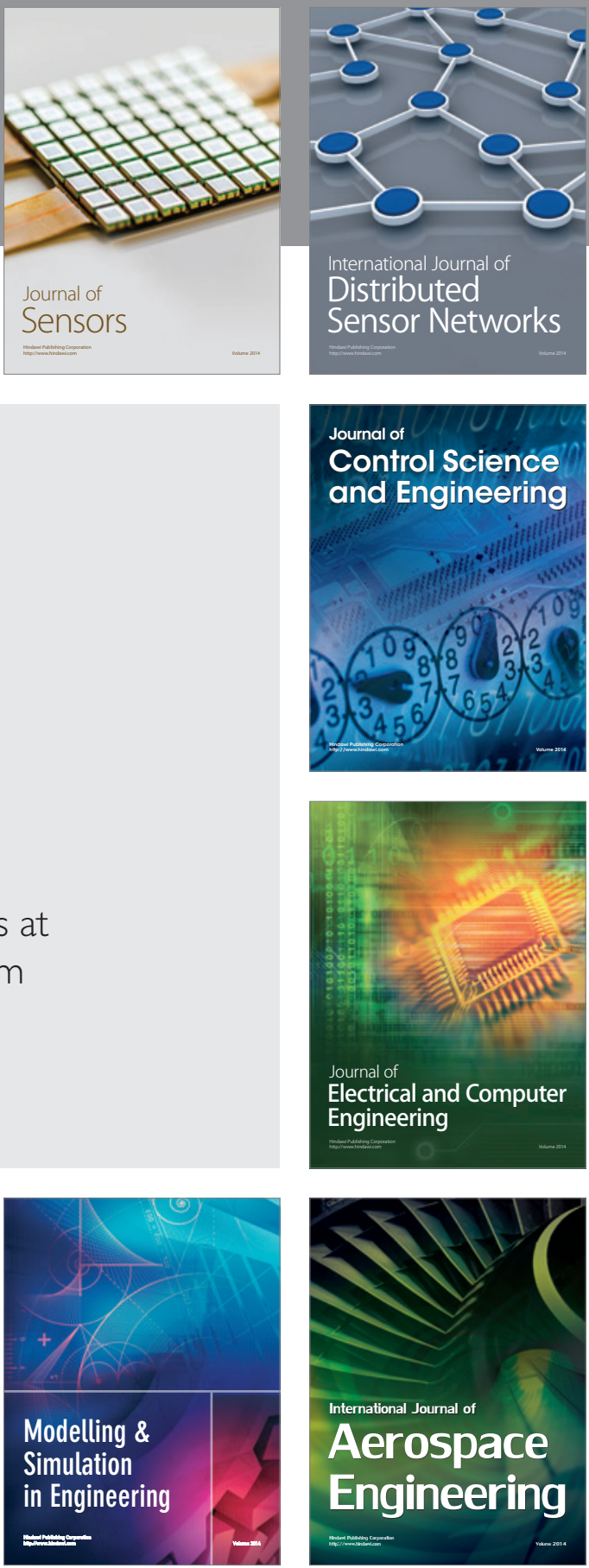

Journal of

Control Science

and Engineering
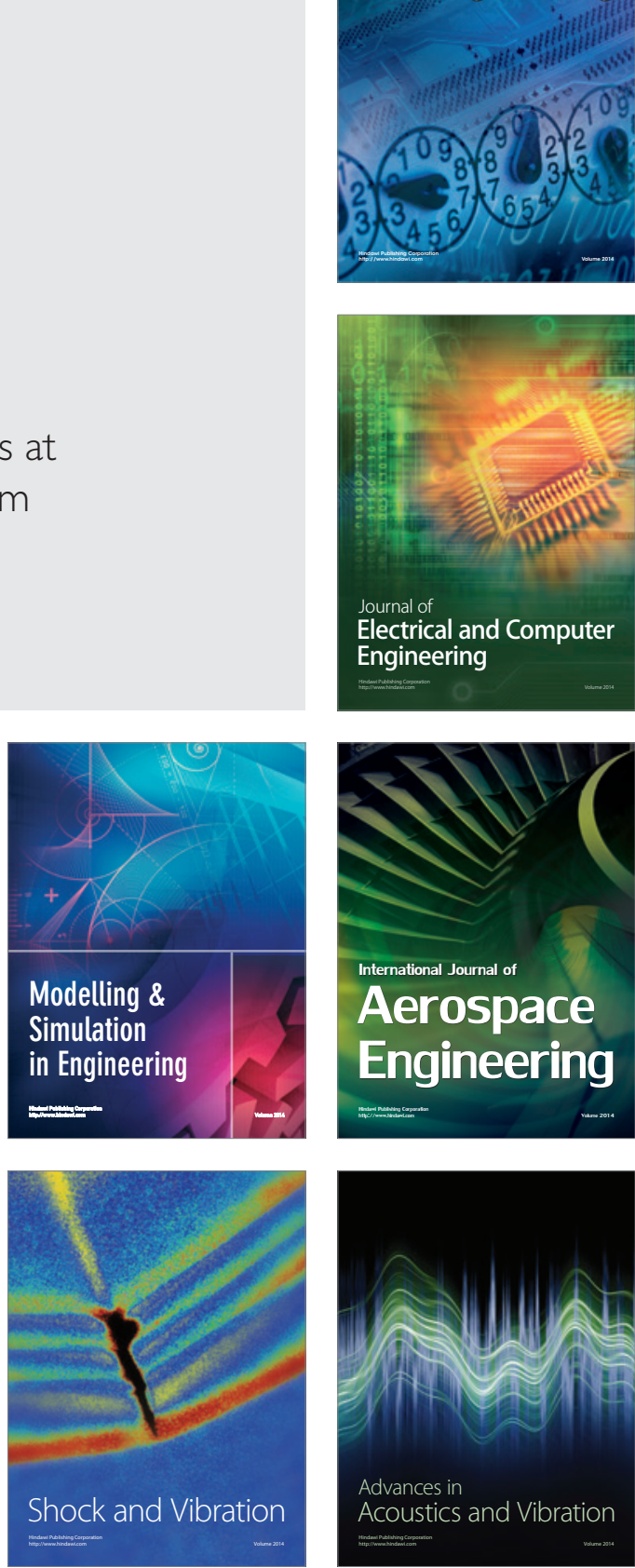